\title{
Contextualización teórica e histórica de la reintegración social y económica de desmovilizados en Colombia
}

\section{Fecha de recepción: 6 de abril de 2010 - Aprobación: 14 de octubre de 2010}

\section{Saridalia Giraldo Gómez}

\section{Resumen}

Este artículo es el resultado de la primera parte de la investigación titulada "Análisis de la política pública de reintegración social y económica en Bogotá durante 2006-2009". El documento estudia los antecedentes históricos de la política de reintegración social y económica, y compara la implementación de la misma con los presupuestos teóricos del proceso.

Para lograr su objetivo, en la primera parte presenta una breve contextualización del conflicto armado en Colombia; posteriormente hace una referencia cronológica de los proceso de reintegración, con especial énfasis en aquellos desarrollados a partir de 2002; en la tercera, realiza un análisis teórico del proceso de desarme, desmovilización y reintegración (DDR); y finalmente concluye que la continuidad en las condiciones que generan el conflicto armado colombiano, no permiten en la actualidad llevar a cabo un proceso de reintegración efectivo.

\section{Abstract}

This article is the result of the first part of the research entitled "Analysis of Public Policy on Social and
Economic Reintegration in Bogotá during 2006-2009."

This paper examines the historical background of the policy of social and economic reintegration, and compares its implementation with the theoretical process. To achieve its objective, the first part presents a brief contextualization of the armed conflict in Colombia; then, it makes a time reference for the reintegration process, with particular emphasis on those developed after 2002. The third part makes a theoretical analysis of the Disarmament, Demobilization, and Reintegration process, and finally, it concludes that the continuity of the conditions that generate the Colombian armed conflict does not allow carrying out, at present, a process of effective reintegration.

\section{Palabras clave}

Proceso DDR, reinserción, reintegración, reconciliación, conflicto armado, grupos armados al margen de la ley.

\section{Key Words}

DDR Process, Rehabilitation, Reintegration, Reconciliation, Armed Conflict, Outlaw Armed Groups.

\section{Introducción}

El conflicto armado colombiano, catalogado como una de las confrontaciones más antiguas y complejas que en la actualidad tienen lugar en el mundo, presenta unas caracterís- ticas particulares que impiden su pronta resolución (Salazar, 2004). Primero, la diversidad de actores cuyo accionar es favorecido por el tráfico de drogas ilícitas; segundo, las principales víctimas de este conflicto son los civiles, 
quienes padecen la constante violación de los derechos humanos y el Derecho Internacional Humanitario (DIH); y tercero, la incapacidad del Estado para garantizar seguridad y justicia en todo el territorio nacional.

En primer lugar, en el conflicto colombiano intervienen actores de naturaleza diversa: las fuerzas de seguridad del Estado; los grupos guerrilleros-en especial las Fuerzas Armadas Revolucionarias de Colombia (FARC-EP) y el Ejército de Liberación Nacional (ELN) -, y los grupos paramilitares.

Por una parte, el Estado colombiano es el tercer receptor de ayuda militar estadounidense en el planeta, después de Israel y Egipto (Colombia Solidarity Campaign, 2003), por causa de la lucha contra las drogas iniciada a partir de la década de los ochenta.

En la actualidad, las fuerzas militares colombianas son las más poderosas de la región, con un total de 285.554 efectivos (distribuidos en Ejército, Fuerza Aérea y Armada Nacional), a los cuales se suman 142.000 miembros de la policía (Tamara, 2010).

Los grupos guerrilleros, por su parte, tienen su origen en cuatro aspectos clave: Primero, la reforma agraria prometida desde el gobierno de Alfonso López Pumarejo (1934-1938) y jamás efectuada, aun cuando era necesaria para evitar la desigualdad social. Esta situación fue el resultado de una colonización espontánea y no dirigida por el Estado, que generó la ocupación de una gran cantidad

\section{Reseña de autor \\ Saridalia Giraldo Gómez \\ Politécnico Grancolombiano \\ giraldo.sari@gmail.com}

Abogada y Politóloga de la Universidad del Rosario. Pertenece al grupo de investigación Derecho, Justicia y Globalización del Programa de Derecho del Politécnico Grancolombiano. de tierras por parte del colono más fuerte o más adinerado, y apartó de toda posibilidad a quienes no tuvieron los recursos para hacer producir su reciente propiedad privada (PNud, 2003). Segundo, el asesinato del líder político Jorge Eliécer Gaitán el 9 de abril de 1948, lo cual defraudó a las masas que veían con su llegada a la presidencia, la posibilidad de reivindicar sus derechos sociales. Originó el período conocido como "La Violencia" (PNUD, 2003).

Por otro lado, la existencia del Frente Nacional (pacto de repartición del poder político entre los partidos Liberal y Conservador efectuado entre 1958-1978), contribuyó con la finalización de la confrontación partidista, pero excluyó de la contienda política al Partido Comunista (Fisas, 2009), el cual capitalizó los remanentes de las guerrillas rurales para avanzar hacia la "combinación de todas las formas de lucha” (Prada, 2007, p. 57); y finalmente, la influencia del contexto internacional, representado fundamentalmente por el triunfo de la Revolución Cubana (1959), el cual fomentó la idea de "que el poder solo se podría conseguir a través de la lucha de guerrillas, siguiendo el ejemplo de Cuba, China y Vietnam" (Prada, 2007, p. 56).

Con respecto a los paramilitares es importante aclarar que el proyecto contrainsurgente en Colombia surgió y se fortaleció, primero por medio de grupos de autodefensas amparados por el Estado para, posteriormente, transformarse en grupos paramilitares al servicio de elites regionales y organizaciones de narcotraficantes, hasta consolidarse como un importante actor con amplio poder político, económico y social en el marco de la confrontación armada interna, el cual inició un proceso de negociación y desmovilización en 2003 (Echeverría, 2007). 
De acuerdo con lo expuesto por Romero (2003), tres factores de índole política contribuyeron a la trasformación de las autodefensas en grupos paramilitares: el proceso de descentralización política y administrativa iniciado a partir de la segunda mitad de los años 1980, la apertura política derivada de la elección popular de alcaldes y gobernadores en 1988, y el contexto de las negociaciones de paz adelantadas por el gobierno de Belisario Betancur con las guerrillas.

Por su parte Alejandro Reyes sostiene que el solo anuncio de la paz pactada fue un chorro de agua fría para el ejército, que sintió frenado su impulso bélico justo cuando creía tener la victoria en sus manos (...) Las brigadas y batallones en áreas guerrilleras comenzaron a organizar el apoyo de los propietarios locales para crear grupos de autodefensa (Reyes Posada, 2004).

En el mismo sentido, Alejandro Reyes Posada (2004) agrega la poca influencia y cooperación entre el Estado y las elites regionales. Por una parte, el Estado no asesoró a dichas elites en cuanto a la forma democrática como debían enfrentar los problemas. Por otra, las elites regionales sentían desconfianza ante un Estado que no lograba contrarrestar la ofensiva guerrillera.

En segundo lugar, las principales víctimas del conflicto son "decenas de miles" de civiles que a diario son amenazados o corren el riesgo de ser reclutados por los grupos armados al margen de la ley (GAML) (CICR, 2009). Asimismo, existe una constante violación de los derechos humanos y el DiH. De acuerdo con la Acnur, Colombia es el país con mayor número de desplazados en el mundo (más de 3 millones en 2007 y 552.000 refugiados) (Acnur, 2008).

En tercer lugar, la limitada presencia estatal en algunas áreas del territorio nacional ha generado la aparición de órdenes paraestatales, en los cuales los GAML imponen "seguridad" y “justicia”. Al respecto, Mauricio Romero, para caracterizar la situación vivida durante la última década del siglo XX, expresa:

El noroeste del país, dominado por grupos contrainsurgentes; la zona andina y central, controlada por las Fuerzas Armadas constitucionales, y el sur y sureste, esta última una zona escasamente poblada donde las FARC tiene una significativa influencia (Romero, 2003, p. 117).

Dadas las circunstancias descritas, el conflicto armado se ha convertido en la principal problemática que ha tenido que afrontar el país durante las últimas tres décadas. Para superarlo, como se verá a continuación, los sucesivos gobiernos lo han incluido en sus agendas políticas, las cuales de acuerdo con el momento, contemplaron múltiples mecanismos para lograr la reinserción de los desmovilizados resultantes de los acuerdos con los GAML.

\section{Contextualización histórica:procesos de desarme, desmovilización y reintegración (DDR) en Colombia}

En este apartado se presentan algunas características de la reinserción en Colombia desde la década de los ochenta. No obstante, es conveniente aclarar que este ejercicio se realiza con el fin de exponer los antecedentes del proceso de reintegración actual, mas no para definirlo puesto que no son comparables.

En efecto, según la Fundación Ideas para la Paz, el proceso DDR, que ha liderado el presidente Álvaro Uribe Vélez se diferencia con los procesos ocurridos principalmente a comienzos de la década de los noventa, debido a que aquellos acogieron a una cifra de ex combatientes inferior al 15\% del número de los actuales desmovilizados; beneficiaron solo a guerrilleros o insurgentes que conta-
Dadas las circunstancias descritas, el conflicto armado se ha convertido en la principal problemática que ha tenido que afrontar el país durante las últimas tres décadas. 
ban con un estatus político; otorgaron suficientes garantías políticas al ser guiados por la favorabilidad; ejecutaron proyectos para el desarrollo económico regional en las áreas de influencia guerrillera, como factores clave para la reinserción; y fueron fuertemente respaldados por la comunidades de acogida. (Giha, Morales y Arias, 2006).

En este orden de ideas, si bien existen algunos referentes de procesos de desmovilización de grupos alzados en armas durante la década de los cincuenta, los antecedentes más antiguos de alternativas de reinserción de ex combatientes, en el contexto de procesos de paz, se dan a partir de los ochenta, con la llegada al poder de Belisario Betancourt Cuartas (1982-1986), quien expidió la Ley 35 del 19 de noviembre de 1982 (Congreso de la República de Colombia, 1982), por medio de la cual se crearon mecanismos para la reincorporación de guerrilleros que dejaran las armas. Si bien esta iniciativa permitió una tregua con varios de los grupos insurgentes, el proceso finalizó sin alcanzar un acuerdo el 6 de noviembre de 1985, con la trágica toma del Palacio de Justicia por parte del Movimiento 19 de Abril (M-19).

Posteriormente, durante el mandato de Virgilio Barco Vargas (1986-1990), en el marco de los acuerdos de paz suscritos con el Partido Revolucionario de los Trabajadores (PRT), el Ejército Popular de Liberación (EPL), el Quintín Lame y el Movimiento 19 de Abril, (M-19), se estableció un nuevo modelo de reincorporación a la vida civil, por medio de la Consejería Presidencial para la Reconciliación, Normalización y Rehabilitación. Este modelo se basó en tres aspectos: (a) la normalización, que se refería a las garantías y principios de favorabilidad para los des- movilizados; (b) la rehabilitación, que se centraba en las poblaciones afectadas por el conflicto; y (c) la reconciliación, en el que se trataba la reinserción de ex combatientes (Plazas Niño, 2006).

El gobierno de César Gaviria Trujillo (1990-1994), continuó con el proceso mediante la expedición del Decreto 2884 de 1991, que creó el programa presidencial para la reinserción, del cual se hicieron beneficiarios según cifras oficiales más de 3.287 (Plazas Niño, 2006, p. 271-336) desmovilizados (la gran mayoría pertenecientes al M-19). Durante este gobierno también fue promulgado el Decreto 1385 de 1994, que sigue siendo una pieza clave en los procesos de reinserción actual. Mediante el mismo, se consideran por primera vez beneficios para los desmovilizados individualmente y se crea el Comité Operativo para la Dejación de Armas (CODA).

Durante las administraciones de Ernesto Samper Pizano (1994-1998) y Andrés Pastrana Arango (1998-2002), el proceso de reinserción en Colombia sufrió una desaceleración producto de los limitados logros en materia de acuerdos de paz con los GAML. En el Gobierno Samper, la política de reinserción estuvo en manos de la Dirección General para la Reinserción, organismo adscrito al Ministerio del Interior. Por su parte, durante el mandato de Pastrana, la Red de Solidaridad Social fue la encargada de acoger a los desmovilizados individuales en el marco del Decreto 1385 de 1994 (Plazas Niño, 2006). Aunque se desmovilizaron 2.505 combatientes entre 1998 y 2002, el programa fue muy limitado y no adquirió gran importancia nacional (Méndezy Rivas, 2008).

Luego de esta breve revisión de los procesos de reinserción, se puede concluir, que la 
política de reinserción durante los años 1980 y 1990, en palabras de la Fundación Cultura Democrática,

tuvo modificaciones en cada gobierno y respondió a las exigencias del mismo proceso. Estuvo marcada por los vaivenes de las negociaciones de paz y el tiempo jugó como referente, pues progresivamente perdió importancia (Plazas Niño, 2006).

\subsection{Programa de reincorporación alavida civil: cambiala perspectiva}

Con la llegada a la Presidencia de Álvaro Uribe Vélez en 2002, se dio un viraje a las políticas de paz manejadas durante los últimos veinte años (Ortiz y Arias, 2007). Por una parte, se reforzó el pie de lucha contra los grupos guerrilleros, con el fin de diezmarlos militar y moralmente en el marco de una estrategia para lograr la movilización individual de los combatientes. Por otro lado, a diferencia de los anteriores gobiernos, se consolidó un proceso de negociación con los grupos paramilitares confederados en las AUC (Pardo, 2007).

En desarrollo de la Ley 782 de 2002 (Congreso de la República de Colombia, 2002), y con el fin de cumplir lo propuesto en los cien puntos del manifiesto democrático (Uribe Vélez, 2002) y en el Plan Nacional de Desarrollo 2002-2006 Hacia un Estado comunitario (Congreso de la República de Colombia, 2003), el gobierno crea el Decreto 128 de 2002, que regula la política de reincorporación a la vida civil y en el marco de este, el Decreto 200 de 2003, con el cual se crea el programa para la reincorporación a la vida civil de ex combatientes y alzados en armas (PRVC), adscrito al Ministerio del Interior, y coordinado por la Comisión Intersectorial para la Reincorporación a la Vida Civil (Ministerio del Interior y de Justicia, 2004), fue el organismo encargado por el gobierno para adelantar el proceso de reinserción social y económica de los ex combatientes. En un principio, apoyó únicamente a los desmovilizados individualmente, que ingresaban al programa por su propia voluntad. Sin embargo, con el avance de las negociaciones entre el gobierno nacional y las AUC, amplió sus funciones para atender a los combatientes desmovilizados colectivamente, fruto de dicho proceso.

A pesar de la creciente oleada de desmovilizaciones individuales generada a partir de 2002 (según la Fundación Ideas para la Paz, FIP, 730 personas se desmovilizaron individualmente en 2002, 2.538 en 2003 y 2.972 en 2004) (Méndez y Rivas, 2008, p. 15), el programa no tuvo gran protagonismo durante el primer año de funcionamiento. Sin embargo, en una coyuntura de continuas desmovilizaciones colectivas, el PRVC estuvo sujeto a múltiples críticas por parte de la opinión pública, que se centraron en los siguientes aspectos: la estructura del PRVC; los procedimientos para acceder a los beneficios del programa y la ausencia de coordinación institucional, tanto horizontal como vertical; y el trato diferencial brindado a los desmovilizados individuales y colectivos en la entrega de la ayuda humanitaria.

En primer lugar, las críticas se relacionaban con problemas estructurales del programa, puesto que había sido concebido como una pequeña oficina, con limitados recursos humanos y presupuestales (Méndez y Rivas, 2008), que desbordaba su capacidad para atender a 1.200 desmovilizados individuales y 31.687 colectivos (Rivas, Méndez, Arias, 2007).

En segundo lugar, en lo referente al acceso al programa y a la ausencia de coordinación institucional, mientras que los desmoviliza-
A pesar de la creciente oleada de desmovilizaciones individuales generada a partir de 2002 (según la Fundación Ideas para la Paz, FIP, 730 personas se desmovilizaron individualmente en 2002, 2.538 en $2003 y$ 2.972 en 2004) 
dos individuales, una vez el CODA certificaba la pertenencia del ex combatiente a un GAML y su voluntad de abandonarlo, eran recibidos por el programa de atención humanitaria al desmovilizado del Ministerio de Defensa (PAHD), que posteriormente los remitía al programa; por su parte, los desmovilizados colectivos, en lo relacionado con el desarme y la desmovilización, eran atendidos por la Oficina del Alto Comisionado para la Paz, quien a través de lista entregada por los representantes de los GAML, ratificaba a las personas que podían acceder al PRVC.

La multiplicidad de organizaciones involucradas en el proceso y la deficiente coordinación horizontal, conllevó el desorden operativo. En este sentido, surgieron fuertes críticas en lo concerniente al número real de desmovilizados de las Auc: al inicio de la administración Uribe, existían datos de alrededor de 12.000 hombres pertenecientes a los grupos contrainsurgentes, pero en 2006, los desmovilizados colectivos (como resultado del proceso de negociación) superaban los 31.000. Al respecto, la Comisión Interamericana de Derechos Humanos (CIDH), señala que

Hubo un incentivo generado por los jefes, para que personas no combatientes se presentaran como miembros de un bloque, en tanto se planteaba la posibilidad de que el (la) no combatiente quedara vinculado a un proceso, en el cual, seria indultado y rápidamente se haría acreedor a un apoyo económico por parte del gobierno (...) de lo cual, debía entregar parte a quien hizo la invitación, como contraprestación por vincularlo al proceso (...). Los desmovilizados hacían parte de los "frentes de apoyo social” de los grupos paramilitares, más que de sus propias filas (Mejía Quintana, 2008, p. 95).

Esta situación ha sido calificada como una falta de compromiso (Mejía Quintana, 2008) por parte del gobierno nacional, para verificar si las personas que accedían al proceso eran realmente miembros de los grupos paramilitares.

Por otro lado, durante 2002 y 2006 , el programa también fue cuestionado por la falta de articulación entre el gobierno nacional y los gobiernos locales (Fundación Ideas para la Paz, 2005), puesto que el grueso de la responsabilidad para lograr la reinserción de los desmovilizados recaía sobre los segundos, a pesar de que la política había sido diseñada y creada por el gobierno nacional sin la participación de las regiones. En esta dirección, la Fundación Ideas para la Paz canaliza los planteamientos de los funcionarios en las regiones, quienes se preguntaban “ ¿ por qué hemos de cargar con la responsabilidad política del éxito de la reinserción en nuestros territorios cuando es una política ideada e impuesta por el Gobierno nacional?" (Fundación Ideas para la Paz, 2005, p. 2).

En tercer lugar, también se presentaron críticas con respecto al tratamiento diferencial de los desmovilizados en el acceso a la ayuda humanitaria. En efecto, los colectivos recibían 358.000 pesos de auxilio económico por un término inicial de dieciocho meses, mientras que los desmovilizados individuales accedían hasta a 895.000 pesos por un periodo de 24 meses (Ministerio del Interior Colombia, PRVC, 2006). Así mismo, mientras que los desmovilizados colectivamente recibían 2 millones de pesos para realizar sus proyectos productivos coordinados por la Oficina del Alto Comisionado para la Paz, los individuales recibían hasta 8 millones dirigidos por el PRVC (Méndez y Rivas, 2008).

De acuerdo con los anteriores planeamientos, las críticas y los errores cometidos durante 
la aplicación de la política de reincorporación a la vida civil, llevaron al gobierno nacional a cambiar la perspectiva y la forma de llevar a cabo el DDR en Colombia. Así, a partir de 2006, el Estado colombiano se abanderó de la "R" de reintegración (Specker, 2008) y no de reinserción o reincorporación como hasta aquel entonces se había manejado. En la siguiente sección, se expondrá el significado de este cambio semántico y las transformaciones generadas a partir de la implementación de la reintegración social y económica.

\subsection{Apuesta por la reintegración:Alta Consejería parala Reintegración Social y Económica}

Las problemáticas expuestas en términos de planeación y ejecución de la política de reincorporación desarrollada por el PRVC, llevaron a que el gobierno replanteará el proceso de desarme, desmovilización y reintegración (DDR) en Colombia. Así, en el Plan Nacional de Desarrollo 2006-2010 Estado comunitario: desarrollo para todos (Congreso de la República de Colombia, 2007), se expresó por primera vez la necesidad de "reintegrar a cambio de reinsertar", lo cual evidenció un giro, no solo semántico sino además de visión y objetivo con respecto al tratamiento de los desmovilizados y la forma de lograr su inclusión social.

En desarrollo de esta perspectiva, el 7 de septiembre de 2006 se expidió el Decreto 3043 mediante el cual se creó la Alta Consejería para la Reintegración Social y Económica (ACR) de personas y grupos alzados en armas, institución vinculada directamente a la Presidencia de la República, que asumió las funciones desa- rrolladas por el PRVC, sumadas a aquellas que resultarían del nuevo objetivo de reintegrar.

De igual modo, el decreto en mención con el ánimo de aclarar la nueva visión del proceso de reintegración, entiende por reintegración la totalidad de los procesos asociados con la reinserción, reincorporación y estabilización social y económica de menores desvinculados y de adultos desmovilizados voluntariamente de manera individual y colectiva. Estos procesos contemplan de manera particular la vinculación y aceptación de estas personas en la comunidad que los recibe, además de la participación activa de la sociedad en general en su proceso de inclusión a la vida civil y legal del país (Presidencia de la República de Colombia, 2006a).

De acuerdo con el concepto de reintegración que ha sido expuesto, se vislumbra la aparente intensión del Estado colombiano de avanzar desde un simple proceso de reinserción asistencialista (Pearl, 2006), caracterizado por la entrega de una mensualidad y algunos elementos necesarios para la supervivencia básica de los ex combatientes, hacia una verdadera inclusión de los mismos en las comunidades de acogida, mediante la implementación de mecanismos de reparación y reconciliación consagrados en los estándares internacionales sobre DDR (Presidencia de la República de Colombia y otros, 2009). Sin embargo, esta intensión no ha logrado consolidarse, ya que la llamada "ruta de reintegración", no incluye más elementos que los vinculados con los "paquetes de reinserción". Esta afirmación, es planteada en la tabla 1: 
Tabla 1. Las etapas del DDR en Colombia

\begin{tabular}{|c|c|}
\hline Etapa del proceso & Actividad \\
\hline Desarme & $\begin{array}{l}\text { Los combatientes (en caso de desmovilizarse individualmente) deben acercarse a } \\
\text { - Miembros de la Policía, el Ejército, la Armada o la Fuerza Aérea. } \\
\text { - Alcaldes o gobernadores. } \\
\text { - Jueces o fiscales. } \\
\text { - Representantes de la Procuraduría General de la Nación o la Defensoría del Pueblo. } \\
\text { En el caso de las desmovilizaciones colectivas, estas se realizan de acuerdo con lo } \\
\text { pactado entre la Oficina del Alto Comisionado para la Paz y el GAML. }\end{array}$ \\
\hline \multirow{3}{*}{ Desmovilización } & $\begin{array}{l}\text { El desmovilizado permanece en una unidad militar o de policía, hasta que sea trasladado } \\
\text { a la ciudad asignada. }\end{array}$ \\
\hline & $\begin{array}{l}\text { El programa de atención humanitaria al desmovilizado y el Ministerio de Defensa } \\
\text { Nacional trasladan al desmovilizado a la ciudad que determine el programa para su } \\
\text { ubicación. }\end{array}$ \\
\hline & $\begin{array}{l}\text { El CoDA investiga sobre la identidad del combatiente y determina si debe ser certificado } \\
\text { como tal. } \\
\text { En caso de ser desmovilizados colectivo, la certificación se efectúa por medio de la } \\
\text { Oficina del Alto Comisionado para la Paz. }\end{array}$ \\
\hline \multirow[b]{3}{*}{ Reinserción } & Desmovilizado permanece en Hogares de Paz en la respectiva ciudad. \\
\hline & $\begin{array}{l}\text { El desmovilizado que desea voluntariamente adelantar el proceso debe primero, } \\
\text { suscribir acta de compromiso con el proceso de reintegración; segundo, presentar } \\
\text { copia de la cédula de ciudadanía; y tercero, cumplir con evaluación de criterios para ser } \\
\text { aceptado en el proceso. }\end{array}$ \\
\hline & $\begin{array}{l}\text { Acceso a la ruta de "reintegración" por medio de centros de servicio en las ciudades: } \\
\text { - Atención en salud. } \\
\text { - Educación. Alfabetización, educación básica, secundaria y posible acceso a } \\
\text { - estudios superiores. } \\
\text { - Atención sicosocial del desmovilizado y su familia. } \\
\text { - Eormación para el trabajo. }\end{array}$ \\
\hline $\begin{array}{l}\text { Reintegración } \\
\text { y } \\
\text { reconciliación }\end{array}$ & $\begin{array}{l}\text { Participación en el proyecto "Ser social", en el cual el desmovilizado desarrolla } \\
\text { actividades como reforestación, arreglo de zonas comunes, mientras se prepara para } \\
\text { el trabajo. } \\
\text { Capacitación en las comunidades en aspectos relacionados con los derechos humanos, } \\
\text { resolución pacífica de conflictos, liderazgo, entre otros. }\end{array}$ \\
\hline
\end{tabular}

Fuente: elaborado por Saridalia Giraldo de acuerdo con el análisis de la página web de la Alta Consejería para la Reintegración Social y Económica (Presidencia de la República de Colombia, 2006a) y su confrontación con los lineamientos teóricos del proceso. 
En este sentido, a la ACR se le encargó desarrollar, ejecutar y evaluar la política de reintegración social y económica de ex combatientes tanto individuales como colectivos, al mismo tiempo que se le asignaron las funciones de gestionar ayuda internacional, administrar recursos y coordinar las actividades de las entidades territoriales y de instituciones, tendientes al cumplimiento de la reintegración (Presidencia de la República de Colombia, 2006a).

De acuerdo con datos del programa de atención humanitaria al desmovilizado, entre agosto de 2002 y agosto de 2009, se desmovilizaron 51.510 personas, de los cuales 19.839 lo hicieron en forma individual y 31.671 de manera colectiva. Los departamentos con mayor acogida de desmovilizados son Antioquia, Córdoba, Cesar, Cundinamarca (en especial en Bogotá), Meta y Valle del Cauca (GiAO Fundación Ideas para la Paz, 2009).

Del total de desmovilizados individuales, $65 \%$ pertenecieron a las FARC, 34\% a las autodefensas, $14 \%$ al ELN y $2 \%$ a grupos disidentes. En cuanto al género, los datos muestran un $83 \%$ de hombres frente al $17 \%$ de mujeres que huyen del conflicto (Ejército Nacional de Colombia, 2007). En promedio, el $37 \%$ de ellas tiene hijos y el $42 \%$ son solteras o se encuentran en unión libre. Con respecto a los desmovilizados colectivos resultantes de los acuerdos con las AUC, 28.751 eran hombres y 2.920 mujeres (Policía Nacional de Colombia, 2009).

En lo referente a los niños, según el Instituto Colombiano de Bienestar Familiar (ICBF), 1.015 niños se desmovilizaron con las AUC (Medios para la Paz, 2006). Por su parte, ee forma individual hasta agosto de 2009, 2.738 menores se unieron al proceso
DDR. Sin embargo, se estima que la cifra es mucho mayor, teniendo en cuenta que hacia 2003, el Secretario General de las Naciones Unidas advirtió sobre 14.000 niños involucrados en los diferentes GAML (PNUD, 2006).

Finalmente, para junio de 2009, la ACR dirigía la reintegración de 31.199 ex combatientes, de los cuales 22.269 pertenecieron a grupos paramilitares, 7.259 hicieron parte de las FARC y 1.398 del ELN y otros grupos (Fundación Ideas para la Paz, 2009). De los vinculados al proceso, el $60 \%$ se encuentra entre los 26 y 45 años de edad. Su nivel educativo es muy bajo, lo que explica que el $42 \%$ de los desmovilizados se encuentren cursando la educación básica primaria y el $58 \%$ básica secundaría.

\section{Contextualización teórica: la reinserción y la reintegración en el proceso DoR}

El proceso DDR entendido como desarme, desmovilización y reintegración, ocupa parte importante en los Estados que se encuentran en transición hacia la paz, al conformarse como una herramienta en la etapa posconflicto y la superación del mismo de manera sostenible (Gleichman, Ondenwald, Steenken y Wilkinson, 2004).

De acuerdo con la politóloga Natalia Springer, este es un proceso

multifocal y multidisciplinario mediante el cual se desactiva el pie de fuerza y se neutraliza la capacidad bélica (defensiva y ofensiva), el aparato financiero, las redes de tráfico y apoyo y la estructura general de operaciones de uno o más grupos armados dentro de un conflicto interno (Springer, 2005, p. 61).

De manera más amplia, el DDR pretende aislar las armas de manos de los insurgentes, romper filas de los grupos armados ilegales y
De los vinculados al proceso, el $60 \%$ se encuentra entre los 26 y 45 años de edad. Su nivel educativo es muy bajo, lo que explica que el $42 \%$ de los desmovilizados se encuentren cursando la educación básica primaria y el $58 \%$ básica secundaría. 
luego de un apoyo económico a los antiguos combatientes, hacerlos parte de las estructuras civiles, sociales y económicas. A la vez que constituye una "oportunidad para la construcción de la seguridad, la estabilidad y la consolidación de la paz" (Carames, Fisas y Luz, 2006, p. 5).

El desarme constituye la primera fase del proceso de DDR. Según la Organización de las Naciones Unidas es el proceso de recolección, control y disposición de las armas pequeñas, municiones, explosivos y armamento ligero y pesado en posesión de combatientes e incluso de las comunidades (ONU, 2006).

Por su parte, la desmovilización se lleva a cabo por medio de los siguientes pasos: planificación, campamento, registro, desarme, orientación antes de la desmovilización y licenciamiento absoluto de los entonces ex combatientes (Gleichman, Ondenwald, Steenken y Wilkinson, 2004). Estos pasos son fundamentales en el proceso de DDR y determinante para el éxito de la reintegración, puesto que significa el cambio de la vida militar a la vida civil del ex combatiente, supone la ruptura del comando, control y la capacidad de las fracciones armadas $y$, en consecuencia, constituye el primer paso hacia la reintegración de los antiguos combatientes en las comunidades receptoras (ONU, 2006).

Finalmente, la desmovilización puede ser individual o colectiva:

Tiene que ver con la disolución de la unidad armada, en caso de desmovilización colectiva, o la reducción del número de combatientes en un grupo, en caso de desmovilización individual, como parte de la transición de la guerra a la paz (Cubides Salazar, 2005, p. 2).
La siguiente fase del proceso es la reintegración. Sin embargo, la reinserción es una etapa anterior que ha sido comúnmente confundida. Así, vale la pena esclarecer que la reinserción es una fase de transición entre la desmovilización y la reintegración. La iniciativa de Estocolmo sobre DDR denomina esta etapa como "transitional safety net", al considerarla como el momento en el que el desmovilizado y su familia necesitan de protección social por medio de elementos materiales que cubran sus necesidades básicas, mientras retoma los suficientes valores sociales y las necesarias acciones de la vida civil para lograr el punto de no retorno a la violencia (Ministry of Foreign Affairs Sweden, 2006).

La reinserción implica el traslado de los combatientes a las regiones de su preferencia para efectuar la reintegración y la entrega de "paquetes de reinserción" que incluyen la provisión de alimentación, ropa, asistencia médica, dinero, herramientas de trabajo e incluso el alojamiento temporal de los desmovilizados (Gleichman, Ondenwald, Steenken y Wilkinson, 2004).

Algunos autores como S. Anderlini y C. Conaway, incluyen a la reinserción en el marco del concepto de reintegración, la cual afirman, consta de dos etapas:

La reinserción inicial y la reintegración a largo plazo. La reinserción se refiere al período breve de la llegada del ex combatiente a su antigua residencia o a una nueva comunidad. La reintegración es un proceso mucho más prolongado que pretende asegurar el desarme permanente y una paz sostenible. Esto incluye asistencia para la comunidad y para el ex combatiente durante la difícil transición a la vida civil. Durante esta etapa, los (o las) ex combatientes pueden disfrutar de servicios de búsqueda de empleo, participar en programas de capacitación para ciertos 
oficios, lo mismo que en programas de crédito, becas o rehabilitación (Anderlini y Conaway, 2006, p. 2).

Por su parte, la reintegración, es definida como

El proceso por el cual los excombatientes adquieren estado civil y tienen acceso a formas civiles de trabajo e ingresos. Esencialmente es un proceso social y económico dentro de un período de tiempo indeterminado que tiene lugar principalmente en las comunidades a nivel local. Es parte del desarrollo general de un país y una responsabilidad nacional para lo cual a menudo se necesita de asistencia externa (Gleichman, Ondenwald, Steenken y Wilkinson, 2004).

Asimismo, la reintegración es fundamental para la recuperación del Estado que supera el conflicto, puesto que además de garantizar el no retorno de los ex combatientes a la violencia, permite la generación de lazos de confianza entre ellos y las comunidades de acogida (Weinstein y Humphereys, 2005). Desde esta perspectiva, la participación de las comunidades es primordial en el proceso, puesto que su apoyo puede contribuir a la vinculación social y económica, y a la consecuente aceptación e inclusión de los ex combatientes (Theidon, 2009, p. 11, 12).

En este sentido, la Contribución de Cartagena al desarme, desmovilización y reintegración, establece que esta última

debe ser integrada a políticas que buscan generar desarrollo social y económico, justicia, visibilización y reparación de víctimas, y seguridad a todos los ciudadanos dentro de un marco democrático (Presidencia de la República de Colombia y otros, 2009).

El gobierno nacional en el documento Conpes 3554 (2008) de "Política nacional de reintegración social y económica para personas y grupos armados ilegales", plantea que la reintegración es un proceso por medio del cual, el desmovilizado adquiere un estatus civil y consigue un empleo e ingreso económico de manera sostenible. En este sentido, el gobierno parte de la situación económica para darle significado a la reintegración social.

De lo anterior se desprende que, mientras la reinserción se refiere a un programa de duración corta y específica mediante el cual se brinda asistencia financiera y material únicamente a los ex combatientes, la reintegración constituye un proceso sin fecha límite (duración de mediano y largo plazos que depende de cada individuo), y en el que la participación de las comunidades es fundamental para lograr la adaptación económica, social y política de los antiguos combatientes (Gleichman, Ondenwald, Steenken y Wilkinson, 2004). En este sentido, la reconciliación se constituye en una etapa del proceso de reintegración y, por tanto, es esencial para el logro de la misma.

La reconciliación es un proceso social que se produce en la etapa del posconflicto y de construcción de la paz, mediante el cual se pretende un cambio de emociones que reconozcan mutuamente un sufrimiento pasado y la necesidad de superar las actitudes y comportamientos destructivos, dando paso a relaciones constructivas y pacificas de una manera sostenible (Brouneus, 2008).

En palabras de Humphereys y Weinstein (2007), la reconciliación es fundamental para lograr la reintegración y la salida del conflicto armado, debido a que los ex combatientes y sus familias requieren de la aceptación de las comunidades de acogida, quienes adquieren una posición dominante
La reconciliación es un proceso social que se produce en la etapa del posconflicto $y$ deconstrucción de la paz, mediante el cual se pretende un cambio de emociones que reconozcan mutuamente un sufrimiento pasado y la necesidad de superar las actitudes $y$ comportamientos destructivos, dando paso a relaciones constructivas y pacificas de una manera sostenible 
Las deficiencias para el logro de la reintegración, no son culpa de las instituciones que lideran el proceso, sino de la existencia de un conflicto armado $(y$, principalmente, de las causas que lo generan), que todavía no permite construir un escenario de paz. sobre la decisión de reintegrarlos a la sociedad civil.

En esta dirección, la Iniciativa de Estocolmo plantea la necesidad de vincular el proceso DDR con los derechos humanos y la justicia transicional puesto que deben restablecerse las relaciones de paz, entre la comunidad receptora y el desmovilizado, para lograr la convivencia y superar el conflicto armado (Ministry of Foreign Affairs Sweden, 2006). Así, se podrá hablar de reintegración una vez sea superada la fase de la reconciliación y la sociedad no solo incluya a los desmovilizados en las actividades sociales y económicas, sino que incluso supere las prácticas de estigmatización.

\section{Conclusión}

En síntesis, de acuerdo con lo expuesto, es posible afirmar que la reintegración es un proceso que no se agota en un solo momento sino que por el contrario requiere de una serie de pasos entrelazados que incluyen previamente el desarme, la desmovilización y la reinserción, y que principalmente se consolida con la reconciliación.

Con la creación del PRVC, la solución adoptada para guiar el proceso fue la rein- serción, con la cual se instauró una salida asistencialista que no era suficiente para superar el problema. Posteriormente, hacia 2006 y en la actualidad con la ACR, a pesar de proyectar la reintegración para guiar el proceso, difícilmente se ha superado la fase de entregar "paquetes de reinserción".

En Colombia, la política nacional aún no ha logrado consolidar un proceso de reintegración, puesto que este prevé la inclusión del desmovilizado en los ámbitos económico, social y político desde la óptica de la reconciliación. Sin embargo, el trabajo con las comunidades de acogida aún es muy deficiente, en parte porque las tareas con la sociedad son limitadas, y en parte porque los colombianos rechazan a los desmovilizados (Pearl, 2008). Pero, ¿cómo exigir reconciliación a una comunidad que aún no conoce el fin de las hostilidades y la construcción de una paz sostenible?

Las deficiencias para el logro de la reintegración, no son culpa de las instituciones que lideran el proceso, sino de la existencia de un conflicto armado (y, principalmente, de las causas que lo generan), que todavía no permite construir un escenario de paz.

\section{Bibliografía}

1. Alto Comisionado de las Naciones Unidas para Refugiados (Acnur). (2008, 17 de junio). Colombia es el país del mundo con mayor número de desplazados. En Caracol Noticias [en línea] Disponible en: http://www.caracol.com.co/nota. aspx?id=615947 (consulta: 4 de diciembre de 2009).

2. Anderlini, S.; Conaway, C. (2006). Desarme, desmovilización y reintegración. [en línea] Disponible en: http://www.reintegracion.gov.co/alta_consejeria/pdf_ normatividad/sobre/Desarme_desmovilizacion.pdf (consulta: 10 de octubre de 2009).

3. Brouneus, K. (2008). Rethinking Reconciliation Concepts, Methods and an Empirical Study of Truth Telling and Psychological Health in Rwanda. Uppsala: Universidad de Uppsala. Department of Peace and Conflict Research. 
4. Carames, A.; Fisas, V.; \& Luz, D. (2006). Análisis de los programas de desarme, desmovilización y reintegración (DDR) existentes en el mundo durante 2005. Quito: Escuela de Cultura de Paz.

5. Colombia Solidarity Campaign. (2003). Uribe's yes to War - The People Say No. Reino Unido: Colombia Solidarity Campaign. Disponible en: http://www.colombiasolidarity. org.uk/content/view/30/54/.

6. Comité Internacional de la Cruz Roja (CICR). (2009, 15 de abril). Población civil es la primera víctima del conflicto armado. En Caracol Noticias. [en línea] Disponible en: http:/www.caracoltv.com/noticias/nacion/articulo135616-poblacion-civil-laprimera-victima-del-conflicto-armado (consulta: 4 de diciembre de 2009).

7. Congreso de la República de Colombia. (2007). Ley 1151 de 2007. Por la cual se aprueba el Plan Nacional de Desarrollo 2006-2010 Estado Comunitario: desarrollo para todos. Bogotá.

8. Congreso de la República de Colombia. (1982). Ley 35 de 1982. Por la cual se decreta amnistía y se dictan normas tendientes al restablecimiento y preservación de la paz. Bogotá.

9. Congreso de la República de Colombia. (2003). Ley 812 de 2003. Por la cual se aprueba el Plan Nacional de Desarrollo 2003-2006. Hacia un Estado comunitario. Bogotá.

10. Congreso de la República de Colombia. (2002). Ley 782 de 2002. Por medio de la cual se prórroga la vigencia de la Ley 418 de 1997, prorrogada y modificada por la Ley 548 de 1999 y se modifican algunas de sus disposiciones. Bogotá: Diario Oficial (45043).

11. Consejo Nacional de Política Económica y Social (Conpes). (2008). Política nacional de reintegración social y económica para personas y grupos armados Ilegales. Conpes 3554. Bogotá: DNP.

12. Cubides Salazar, OM. (2005). Experiencias internacionales de procesos de desarme, desmobilización y reinserción DDR: lecciones y aprendizajes. Bogotá: Fundación Seguridad y Democracia.

13. Echeverría, AE. (2007, primer semestre). Las limitaciones que enfrentaría la instauración de una Comisión de la Verdad, en el proceso de tránsito hacia la paz entre el Estado y las Autodefensas Unidas de Colombia (AUC). Desafíos, 279 -315.

14. Ejército Nacional de Colombia. (2007, 12 de mayo). En los últimos cuatro años se han desmovilizado 1893 mujeres. En Ejército Nacional de Colombia [en línea] Disponible en: de http://www.ejercito.mil.co/?idcategoria=191777 (consulta: 5 de diciembre de 2009),

15. Fisas, V. (2009). Anuario procesos de paz 2009. Barcelona: Escuela de Cultura de Paz.

16. Fundación Ideas para la Paz. (2009). Narcotráfico y rearme amenazan la reintegración de desmovilizados. En Verdad Abierta.com Paramilitares y Conflicto Armado en Colombia (en línea] Disponible en: http://www.ideaspaz.org/secciones/verdad_abierta/ pdf_articulos_verdad_abierta_\%20fip/narcotrafico_rearme_amenazan_\%20 reintegracion_desmovilizados.pdf (consulta: 10 de diciembre de 2009).

17. Fundación Ideas para la Paz. (2005, noviembre). Reinserción: primeras conclusiones. En Siguiendo el conflicto: hechos y análisis [en línea] Disponible en: http://www. 
ideaspaz.org/publicaciones/download/boletin_conflicto38.pdf (consulta: julio de 2009).

18. GiaO Fundación Ideas para la Paz. (2009, 31 de agosto). Estadísticas sobre reinserción en Colombia. En Verdad Abierta [en línea] Disponible en: www. verdadabierta.com/.../10-desmovilizacin-y-desarme?...506\%3Aestadisticas-sobre reinsercion-en-colombia...2009 (con sulta: 18 de noviembre de 2009).

19. Giha, Y.; Morales, L.; \& Arias, I. (2006). Evaluación de los procesos de reinserción colectivos de la década de los noventa. Bogotá: Fundación Ideas para la Paz.

20. Gleichman, C.; Ondenwald, M.; Steenken, K.; \& Wilkinson, A. (2004). Desarme, desmovilización y reintegración. Guía teórica y práctica. Frankfurt: Imprenta Hassmuller.

21. Humphreys, M., \& Weinstein, J. (2007). Demobilization and Reintegration. Journal of Conflict Resolution. Sage Publicaciones Volumen 51 , 531-567.

22. Medios para la Paz. (2006). Menores combatientes: una realidad que se esconde. En Medios para la Paz [en línea] Disponible en: www.mediosparalapaz. org?idcategoria $=829$ (consulta: 10 de octubre de 2009).

23. Mejía Quintana, JO. (2008). El fenómeno paramilitar: reflexiones, apuntes y discusiones. En Universidad Nacional de Colombia, Paramilitarismo, desmovilización y reinserción. La ley de justicia y paz y sus implicaciones en la cultura política, la ciudadanía y la democracia en Colombia. Bogotá: Universidad Nacional de Colombia, p. 83-114).

24. Méndez, ML.; \& Rivas, A. (2008). Alternativas de generación de ingresos para desmovilizados: el programa de reinserción a la vida civil y la Alta Consejería para la Reinserción. Bogotá: Fundación Ideas para la Paz.

25. Ministerio del Interior y de Justicia Colombia (2004). Decreto 1262 de 2004. Por el cual se crea una Comisión Intersectorial para la Reincorporación a la Vida Civil de Personas y Grupos Alzados en Armas. Bogotá.

26. Ministerio del Interior y de Justicia Colombia. Programa para la reincorporación a la vida civil. (2006). Política de reincorporación a la vida civil 2003-2006, una mirada institucional. En T. Contreras Rolón; M. Jaramillo de Botero; \& A. Picón Amaya. Bogotá. Disponible en :www.verdadabierta.com/.../10-desmovilizacin-ydesarme?...vida-civil-ministerio-del-interior - .

27. Ministry of Foreign Affairs Sweden. (2006). Stockholm Initiative on Disarmament, Demobilisation and Reintegration. Final Report. Estocolmo: Sundh, Lena; Samuelsson, Jens Editores.

28. Organización de las Naciones Unidas (ONU). (2006, Sixtieth Session). Disarmament, Demobilization and Reintegration. En Organización de las Naciones Unidas [en línea] Disponible en: http://www.unddr.org/iddrs/01/20.php (comnsulta: 15 de septiembre de 2009).

29. Ortiz, R.; \& Arias, A., (2007). Nuevos retos en un viejo conflicto: el futuro de la seguridad democrática. En Siguiendo el conflicto: hechos y análisis de la semana. Bogotá: Fundación Ideas para la Paz.

30. Pardo, R. (2007). Fin del paramilitarismo. ¿Es posible su desmonte? Bogotá: Ediciones B. 
31. Pearl, F. (2006, 5 de noviembre). Proceso con los paras esta en su momento más crítico. En Centro Virtual de Noticias [en línea] Disponible en: http://www. mineducacion.gov.co/cvn/1665/fo-article-112079.pdf (consulta: 3 de diciembre de 2009).

32. Pearl, F. (2008, 16 de abril). Video Conferencia de grandes líderes. En Universidad CESA: http://video.google.com.co/videoplay?docid=-6289352809071042609\&ei= cHVsS4STEJLmqgL_9tHqBg\&q=Conferencia+de+Grandes+L\%C3\%ADderes+ Frank+Pearl+\&hl=es\# (consulta: 18 de noviembre de 2009).

33. Plazas Niño, NR. (2006). Política pública de reinserción y protocolo de actuación humanitaria. En AS. Villarraga, La reinserción en Colombia: experiencias, crisis humanitaria y política pública. Bogotá: Fundación Cultura Democrática, Asociación de Constructores de Paz.

34. Policía Nacional de Colombia. Área de Información Delincuencial y Análisis Criminológico. (2009). Desmovilizados colectivos e individuales. Informe Control y Monitoreo. Bogotá: Polícia Nacional de Colombia.

35. Prada, O. (2007). Las cifras del conflicto colombiano. Bogotá: Indepaz.

36. Presidencia de la República de Colombia. (2006a). Decreto 3043 de 2006. Por el cual se crea una Alta Consejería en el Departamento Administrativo de la Presidencia de la República. Bogotá.

37. Presidencia de la República de Colombia. (2006b). Ruta de reintegración. En Alta Consejería para la Reintegración: [en línea] Disponible en: http://www. reintegracion.gov.co/Es/ruta_reintegracion/Paginas/ingreso.aspx (consulta: 23 de abril de 2009).

38. Presidencia de la República de Colombia y otros. (2009). La contribución de Cartagena al desarme, desmovilización y reintegración. Auspiciado por el Banco Mundial [en línea] Disponible en: http://www.reintegracion.gov.co/Es/ prensa/Documents/centro_doc/contribucioncartagenaddr.pdf (consulta: 18 de noviembre de 2009).

39. Programa de las Naciones Unidas para el Desarrollo (PNUD). (2006, julio). Menores combatientes: una realidad que se esconde. En Hechos del callejón. Bogotá: PNUD, 6-8.

40. Programa de las Naciones Unidas para el Desarrollo (PNUD). (2003). El conflicto, callejón con salida. Informe Nacional de Desarrollo Humano Colombia. Bogotá: PNUD, El Malpensante.

41. Reyes Posada, A. (2004). Paramilitarismo en Colombia. Contexto, aliados y consecuencias. En d.E. Instituto, Guerras en Colombia, actores, armas. Bogotá: Universidad Nacional.

42. Rivas, A.; Méndez, ML.; \& Arias, G. (2007). De ex combatientes a ciudadanos: luces y sombras de los nuevos planes de desmovilización y reintegración. Bogotá: Fundación Ideas para la Paz.

43. Romero, M. (2003). Paramilitares y autodefensas 1982-2003. Bogotá: Editorial Planeta.

44. Salazar, G. (2004). Mirada crítica al conflicto armado colombiano. En Acnur. org [en línea] Disponible en: http://www.acnur.org/biblioteca/pdf/3825.pdf (consulta: 16 de octubre de 2009). 
45. Specker, L. (2008). The R phase of DDR Process. An Overview of Key Lessons Learned and Practical Experiences. Amsterdam: Netherlands Institute of International Relations "Clingendael".

46. Springer, N. (2005). Desactivar la guerra. Alternativas audaces para consolidar la paz. Bogotá: Aguilar.

47. Tamara, E. (2010, 12 de febrero). Colombia se transforma en la mayor Fuerza Armada de Suramérica. En: Barómetro Internacional. [en línea] Disponible en: http://www.barometro-internacional.org/index.php?option=com_content\&task $=$ view\&id=515\&Itemid=1 (consulta: 15 de marzo de 2010).

48. Theidon, K. (2009, mayo). Reintegrating Former Combatans in Colombia. En Focus.

49. Uribe Vélez, A. (2002). Manifiesto democrático: cien puntos. [en línea] Disponible en: www.mineducacion.gov.co/1621/articles-85269_archivo_pdf.pdf (consulta: septiembre de 2009).

50. Weinstein, J.; Humphereys, M. (2005). Disentangling the Determinants of Successful Demobilization and Reintegration. Washington DC: Center for GLobal Development. 
$\rightarrow$ DERECHO 


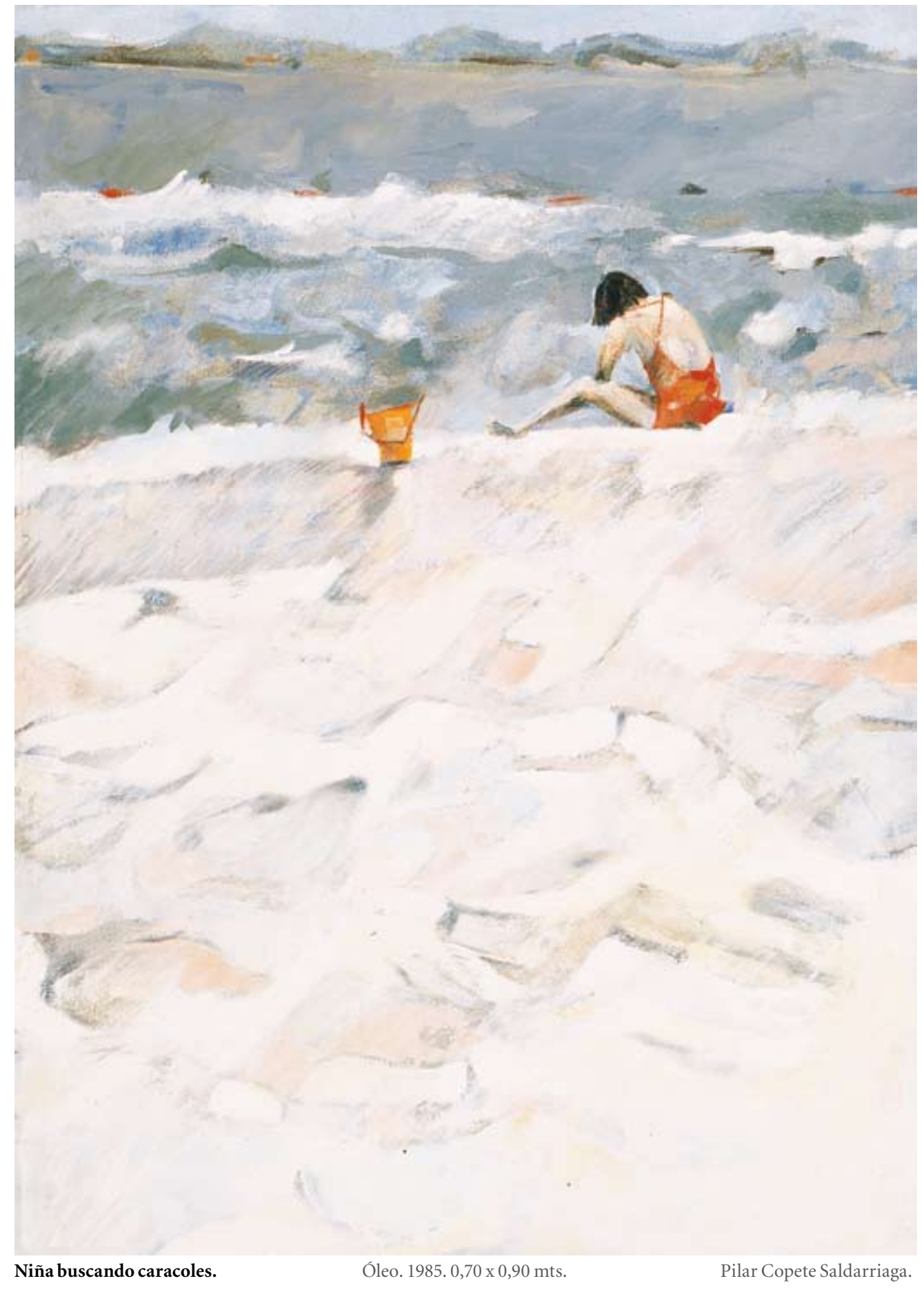

